\title{
Detection of reactor neutrinos with a delayed signal of neutron capture on Hydrogen at RENO
}

\section{Eunhyang Kwon*}

Seoul National University, Gwanak-ro 1, Gwanak-gu, Seoul, Republic of Korea

E-mail: ehkwon1011egmail.com

\begin{abstract}
The RENO experiment has been taking electron antineutrino data from the reactors at Hanbit nuclear power plant(Younggwang, Korea), using two identical detectors since 2011. The RENO experiment has measured using inverse beta decay events where neutrons are captured on Gadolinium $(\mathrm{Gd})$. The result of RENO experimenth as published the results only the Gd-captured analysis 2012 and 2016. However, the neutrons also can be captured by hydrogen. Neutron captured on hydrogen analysis can give independent result from neutron capture on Gd analysis. Hydrogen analysis(nH) has longer time interval of delayed signal than Gd-captured and it contains more accidental background. We have measured number of the inverse beta decay events and the backgrounds even accidental and fast neutrons, Li/He. In RENO experiment, Hydrogen analysis can use the about 2000 days data and we have been preparing the result using 1500 days data
\end{abstract}

The 39th International Conference on High Energy Physics (ICHEP2018)

4-11 July, 2018

Seoul, Korea

${ }^{*}$ Speaker. 


\section{Introduction}

The neutrons from the IBD process can be also captured by Hydrogen. But it has a longer time interval $(\sim 200 \mu \mathrm{s})$ of delayed signal than captured on Gadolinium( $\sim 26 \mu \mathrm{s})$ [四. Hydrogen captured analysis has more backgrounds than Gd captured analysis so that it is important to reduce the background and measure them.

\section{2. backgrounds of Hydrogen captured analysis}

There are three kinds of backgrounds same with $\mathrm{n}-\mathrm{Gd}$ analysis. Accidental background is the coincidence events between prompt and delayed signal. It is the largest portion of n-H analysis Fast neutron background is produced by muons from surrounding rocks and inside detector. $\mathrm{Li} / \mathrm{He}$ backgrounds produced by cosmic muon spallation. The characteristic of accidental backgrounds is large $\Delta R$ and $\Delta T . \Delta R$ within $50 \mathrm{~cm}$ cut and 2-dimensional cut with $\Delta R$ and $\Delta T$ are used to reduce the background. Fast neutron(FN) distribute up to high energy region so Fitting to high energy region $(\sim 60 \mathrm{MeV})$. To estimate the remained $\mathrm{Li} / \mathrm{He}$ background, makes $\mathrm{Li} / \mathrm{He}$ template using muon time difference. In addition to after $\mathrm{Cf}$ contamination, $\mathrm{Cf}$ background should be removed. It has low amount than $\mathrm{n}-\mathrm{Gd}$ analysis and fitting $12 \sim 22 \mathrm{MeV}$ region and estimate the $\mathrm{Cf}$ background with find minimum difference between $\mathrm{FN}+\mathrm{Cf}$ shape and IBD candidates.

\section{IBD and background rate}

Total IBD candidates is 559,844 (near) and 84,817 (far) using about 1500 days data. In near, $1.7 \%$ of accidental background, $0.8 \%$ of fast neutron, $1.7 \%$ of $\mathrm{Li} / \mathrm{He}$ background are estimated and in far, $30 \%$ of accidental, $1.4 \%$ of fast neutron, $2.4 \%$ of $\mathrm{Li} / \mathrm{He}, 0.03 \%$ of $\mathrm{Cf}$ background are estimated.
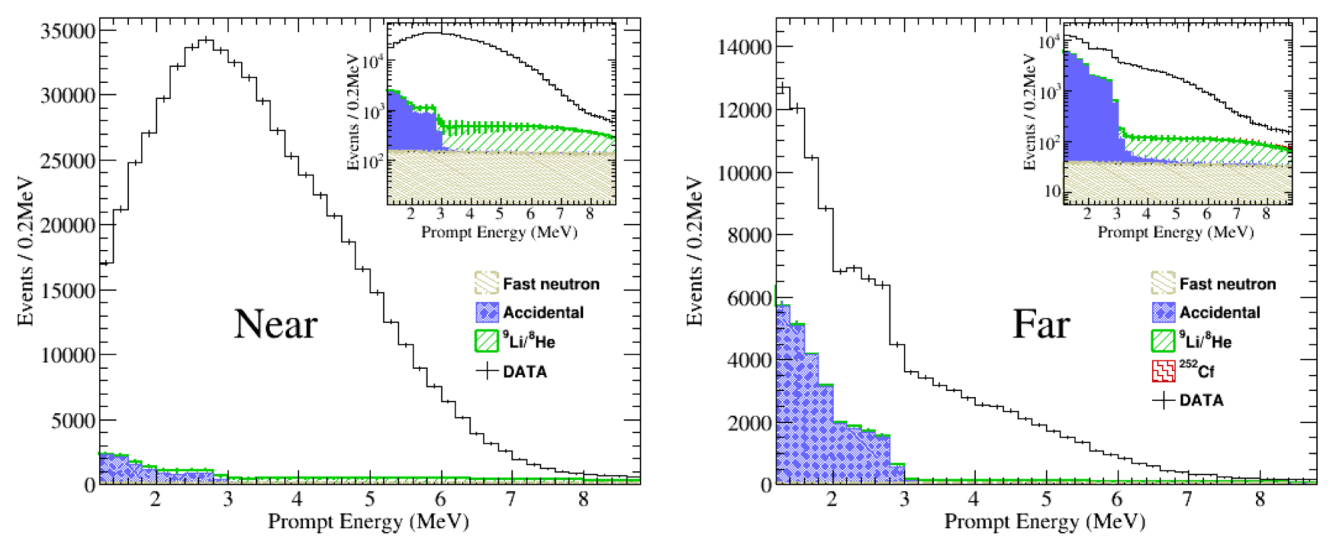

Figure 1: Distribution of IBD candidates and backgrounds. (left) Near and (right) far.

\section{References}

[1] S.H.Seo et al. (RENO Collaboration), Spectral measurement of the electron antineutrino oscillation amplitude and frequency using 500 live days of RENO data, Phys. Rev. D 98, 012002 (2018) 